\title{
CATÁLOGO DE LOS HONGOS AISLADOS EN EL POLVO ACUMULADO EN COLEGIOS DE CÓRDOBA (ESPAÑA)
}

\author{
Julia ANGULO ROMERO, Félix INFANTE GARCIA-PANTÄLEÓN, \\ Ana MEDIAVILLA MOLINA y Eugenio DOMÍNGUEZ VILCHES
}

RESUMEN. Catálogo de los hongos aislados en el polvo acumulado en colegios de Córdoba (España). En este trabajo se ha analizado el polvo de 12 Colegios de E.G.B. de la ciudad de Córdoba, con objeto de identificar, cuantificar y catalogar la micoflora presente en él, así como conocer su frecuencia y estacionalidad. De esta manera hemos aislado 91 táxones, los cuales se relacionan en el presente catálogo, indicando para cada uno de ellos si son potencialmente patogénicos, época del año en la que se han detectado e incidencia en nuestros muestreos.

Palabras clave. Esporas fúngicas, hongos microscópicos, polvo doméstico, España.

ABSTRACT. Checklist of the fungi isolated from school dust in Cordoba (Spain). The micoflora presents in dust of 12 schools of the city of Cordoba have been studied. A check list indicating seasonality, frequency and incidence of the 91 taxa is included.

Key words. Spore fungi, fungi, school-dust, Spain.

\section{INTRODUCCIÓN}

$\mathrm{El}$ «polvo doméstico» es considerado una mezcla compleja que incluye numerosas sustancias inorgánicas y orgánicas de origen animal o vegetal tales como: arena, ceniza, restos de comida, fibras textiles, ácaros, hongos, pólenes, algas, insectos, bacterias, restos dérmicos humanos y de animales, etc., así como productos degradados de todos estos componentes. Inicialmente la presencia fúngica en un hábitat determinado puede deberse a una deposición fortuita y posteriormente producirse una verdadera colonización fúngica.

Los primeros investigadores que demues- tran la presencia de propágulos viables de hongos en el polvo doméstico fueron Davies (1958) y Werff (1958). En 1972 Bronswijk (1972) se refiere por primera vez al polvo doméstico como un ecosistema terrestre más y desde esta perspectiva comienza a ser investigado por distintos autores, señalando éstos la necesidad de hacer mención expresa de la metodología utilizada en el aislamiento y caracterización de los hongos del polvo, ya que la misma condiciona la composición cualitativa y cuantitativa de los hongos detectados (Solomon, 1975). Posteriormente otros autores (Gravesen, 1981; Torres et al., 1982; Duce 
et al., 1986) indican lo importante que desde el punto de vista higiénico/sanitario resulta conocer la distribución de hongos en un determinado hábitat, distribución que según ellos dependería de modificaciones ambientales (sobre todo humedad y temperatura).

Aunque está bien establecida la importancia del polvo como contaminante, hasta la fecha han sido poco frecuentes las investigaciones dedicadas a esclarecer la influencia de los hongos del polvo en lugares de trabajo no industriales, tales como oficinas y/o escuelas (Gravesen etal., 1983, 1986; Mercantini et al., 1983, 1986; Sederberg-Olsen \& Hardt, 1984; Samson, 1985 y Gravesen, 1987). En España los primeros estudios sobre el polvo doméstico se remontan a 1930 en el que Bernton (1930) relaciona los conidios fúngicos con las alergias producidas por el polvo de las casas. Ales et al. (1957) y Jimenez-Diaz et al. (1960), realizan un estudio sobre el contenido de esporas de hongos en el polvo doméstico de ciudades de interior y costeras. Portus et al. (1977), señalan como en ciertos microhábitats los hongos favorecen el desarrollo de especies de ácaros. Dronda (1981), realiza un estudio cualitativo y cuantitativo de los hongos presentes en el polvo doméstico, y compara la micoflora existente en éste en los hogares de sujetos alérgicos y sanos. Un estudio similar es llevado a cabo por Calvo et al. (1982).

Vista la importancia que ha alcanzado en los últimos años el estudio de los problemas en los ambientes de interiores (WHO, 1983), nuestro grupo, que ya venía realizando trabajos sobre este tipo de micoflora (Infante y Domínguez, 1987, 1988; Infante et al., 1987a, 1987b, 1987c), se planteó el estudio de los hongos presentes en el polvo; elegimos doce Colegios de Enseñanza General Básica (E.G.B.) en los que nos propusimos identificar, cuantificar y catalogar la micoflora presente en ellos, así como conocer la frecuencia y estacionalidad de especies potencialmente patogénicas.

\section{METODOLOGÍA}

El estudio se realizó en Córdoba (España) ciudad que posee unos 300.000 habitantes, situada a unos 120 m.s.m. en la falda de Sierra Morena y dividida en dos partes por el río Guadalquivir. Los puntos de muestreo (12 colegios de E.G.B.) fueron elegidos aleatoriamente, pero de manera que estuvieran distribuidos por distintas zonas de la ciudad.

La toma de muestras se realizó en el período correspondiente a dos cursos académicos consecutivos, con una periodicidad quincenal (excepto los meses de vacaciones de verano, julio y agosto). De esta manera, se han obtenido un total de 456 muestras de polvo. La recogida de muestras, se efectuó con una aspiradora portátil adaptada por nosotros para tal fin, que era limpiada con alcohol y sometida a luz ultravioleta después de cada muestreo. El polvo recogido del suelo de un aula (siempre la misma y elegida al azar), se guardaba a temperatura ambiente en bolsas estériles de plástico, selladas y rotuladas convenientemente hasta el momento de su procesado.

La inoculación del polvo se realizó mediante siembra directa $(10 \mathrm{mg})$ en cajas de Petri conteniendo como medio de cultivo agar extracto de malta al $2 \%$, según la fórmula de Blakeslee (Tuite, 1969). Al medio se le añadió una solución de ácido láctico para inhibir el crecimiento bacteriano, posteriormente las placas se incubaban durante 7 días, transcurridos los cuales se procedía al recuento, aislamiento e identificación de las colonias desarrolladas.

\section{CATÁlogo}

En las especies citadas en el presente catálogo, y ordenadas alfabéticamente se comenta: si son potencialmente patogénicas, referencias bibliográficas encontradas para el polvo doméstico, época del año en la que la hemos aislado e incidencia en nuestros 
muestreos (Ocasional, 0-5 meses; Frecuente, 6-8 meses; Permanente, más de 8 meses).

Absidia ramosa (Lindt) Lendner, 1908.

Aislada por Davies (1960) de polvo doméstico. Ocasional en nuestros muestreos (otoño-invierno).

Acremonium alternatum Link per S.F. Gray, Mag. Ges. Naturf. Fr., Berlín, 3: 15, 1809.

Ocasional en nuestros muestreos, la casi totalidad de las colonias se aislaron en otoño del primer año.

Alternaria chartarum Preuss, F. Hoyersw 27, 1854.

Alternaria citri Ellis \& Pierce.

-Stemphylium citri Patterson \& Charles.

Aislada de muestras de polvo doméstico en Barcelona por Dronda (1981). Ocasional en nuestros muestreos, y detectada preferentemente en la primavera.

Alternaria consortiale (Thüm) Hughes, Canad. J. Bot. XXXI: 636, 1953. Skolko.

Stemphylium consortiale (Thüm) Groves \&

Frecuente en nuestros muestreos y aislada preferentemente en la primavera.

Alternaria dendritica (S. da Cam.) Joly, Le Genre Alternaria: 147, 1964.

Stemphylium dendriticum S. da Cam.

Frecuente en nuestros muestreos y sin estacionalidad acusada.

Alternaria oleracea Milbraith, Bot. Gaz. 74: 320, 1922.

A. brassiccicola (Schw) Wiltshire.

Frecuente en nuestros muestreos y sin estacionalidad.

Alternaria sonchi Davies ex Elliott, Bot. Gaz., LXII: 414, 1916.

Identificada como ocasional, sólo se detectó en otoño del primer año.

Alternaria tenuis Nees, Syst. Pilze Schwamme: 72, 1816/17.

A. alternata (Fr.) Keissler.
Citada como agente alergógeno (Bonilla-Soto et al., 1961; Hyde, 1972; D’Amato et al., 1975 y Muñoz y Martin, 1983). Aislada en el polvo doméstico por Davies (1960), Bronswijk \& Sinha (1973), Abdel-Hafez et al. (1986), Horak (1987) y Dronda (1981). Permanente en los muestreos realizados, es la especie de Alternaria de mayor incidencia, presentando los máximos en el mes de junio.

Alternaria tenuissima (Fr.) Wiltshire, Trans. Br. Mycol. Soc. 28: 157, 1933.

Especie de poder patógeno relativamente débil (Joly, 1964). Aislada de polvo doméstico por Abdel-Hafez et al. (1986). Permanente en nuestros muestreos, presenta sus máximos durante los meses de otoño e invierno.

Arthrinium phaeospermum (Corda) Ellis, Mycol. Pap. 103: 8-10,1965.

Papularia phaeosperma (Pers) Höhnel.

Ocasional en los muestreos realizados y detectada en primavera.

Aspergillus alliaceus (Thom \& Church) Fennell \& Warcup, Mycologia, 51: 411-413, 1959.

Ocasional en nuestros muestreos.

Aspergillus candidus Link, Observationes: 16, 1809.

Esta especie ha sido citada en el polvo doméstico por Davies (1960) y Abdel-Hafez et al. (1986). Frecuente en nuestros muestreos, con máximos en el otoño.

Aspergillus carneus (V.Tiegh.) Blochwitz, Thom \& Raper, A. manual of the Aspergilli: 201202,'1945.

Frecuente en nuestros muestreos, tiende a aparecer a finales de invierno y principios de primavera.

Aspergillus eburneo-cremeus Sappa, in Boll. Ist. Orto. Botan. Univ. Torino, 2(1): 80-84, 1954. Ocasional en nuestros muestreos y $\sin$ estacionalidad.

Aspergillus flavipes (Bain \& Sart.) Thom \& Church, The Aspergilli: 155, 1926.

Aislada del polvo doméstico por Abdel-Hafez 
et al. (1986). Ocasional en nuestros muestreos y detectada fundamentalmente durante la primavera del segundo año.

Aspergillus flavus Link, in Observationes: 16, 1809.

Citada por Hyde (1972) como productora de esporosis. Aislada en muestras de polvo doméstico en Londres (Davies, 1960), Paises Bajos (Bronswijk \& Sinha, 1973), Egipto (Abdel-Hafez et al., 1986) y España (Dronda, 1981). Ocasional en nuestros muestreos y detectada durante el mes de octubre del primer año.

Aspergillus fumigatus Fres., Beiträge zur Mykologie: 81, 1863 .

Citada como agente potencialmente patogénico por varios autores (Hyde, 1972; Gregory, 1973; D'Amato et al., 1975; Mullins et al., 1976; Calvo et al., 1978 y Gravesen, 1979). Aislada en muestras de polvo doméstico como indican Davies (1960), Bronswijk \& Sinha (1973), Hirsch \& Sosman (1976), Gravesen (1978), Abdel-Hafez et al. (1986) y Horak (1987). Permanente en nuestros muestreos, destacando los meses de mayo, junio y septiembre del segundo año.

Aspergillus japonicus Saito, Botan. Mag. (Tokyo) 20: 61-63, 1906.

Ocasional en nuestros muestreos.

Aspergillus melleus Yukawa, in J. Coll. Agr. Imp. Univ. Tokyo 1(3): 366, 1911.

Aislada de polvo doméstico (Abdel-Hafez et al., 1986). Creemos que esta especie no había sido citada anteriormente en España. Ocasional en nuestros muestreos.

Aspergillus niger V. Tiegh, Ann. Sci. Nat. Bot., Ser. 5, 8: 240, 1867.

Citada en la bibliografía como potencialmente patogénica (Hyde, 1972; D’Amato et al., 1975; Sandhu et al., 1978; Samson, 1985). Aislado por Gravesen (1978) en el polvo de hogares daneses, Davies (1960) en ingleses, Bronswijk \& Sinha (1973) en holandeses, Abdel-Hafez et al. (1986) en egipcios y Dronda (1981) en españoles. Permanente en nuestros muestreos, presentando varios máximos en el año. Es la especie de la que hemos aislado mayor número de colonias.
Aspergillus niveus Blochwitz, Ann. Mycol. 27(3/ 4): 205-206, 1929.

Encontrada en el polvo doméstico de Barcelona por Dronda (1981). Frecuente en nuestros muestreos y sin estacionalidad.

Aspergillus ochraceus Wilhelm, Inagural

Dissertation, Strasburg: 66, 1877.

Especie potencialmente patogénica (Novey \& Well, 1978). Se ha obtenido a partir de polvo doméstico en Holanda (Bronswijk \& Sinha, 1973), Dinamarca (Gravesen, 1978), Egipto (Abdel-Hafez et al., 1986) y España (Dronda, 1981). Ocasional en nuestros muestreos.

Aspergillus oryzae (Ahlburg) Cohn, Jahresber. Schles. Ges. Vaterl. Kultur, 61: 224, 1884.

Permanente en nuestros muestreos y detectada preferentemente en los meses de septiembre a noviembre.

Aspergillus parasiticus Speare, in Hawaiian Sugar Planters Assoc. Expt. Sta., Pathol. and Physiol. Ser., Bull. 12: 38, 1912.

Especie citada como potencialmente patogénica (Novey \& Well, 1978; Ciegler, 1979 y Chakraverty \& Sinha, 1985). Aislada en el polvo doméstico por Abdel-Hafez et al. (1986). Ocasional en nuestros muestreos.

Aspergillus petrakii Vöros Sydowia, Ann. Mycol., Ser. II, Beihefte 1: 62-63, 1957.

Ocasional en nuestros muestreos. Aislada sólo en el segundo año.

Aspergillus silvaticus Fennell \& Raper, Mycologia, 47: 83-86,1955.

Ocasional en nuestros muestreos.

Aspergillus speluneus Raper \& Fennell, in The Genus Aspergillus: 457-460, 1965.

No tenemos referencias de que esta especie haya sido citada con anterioridad en nuestro país. Ocasional en nuestros muestreos.

Aspergillus sydowi (Bain \& Start) Thom \& Church, The Aspergilli: 147, 1926.

Citada en polvo doméstico por Davies (1960) y Abdel-Hafez et al. (1986). Frecuente en nuestros muestreos, presentando los máximos en la primavera. 
Aspergillus terreus Thom, in Thom \& Church, Am. J. Botany, 5: 85-86, 1918.

Productor de aspergilosis broncopulmonar alérgica (Sandhu et al., 1978). Aislado de muestras de polvo doméstico por Abdel-Hafez et al. (1986). Permanente en nuestros muestreos, presentando los máximos durante el invierno y tambien en abril del segundo año.

Aspergillus ustus (Bainier) Thom \& Church, The Aspergilli: 152, 1926.

Aislada a partir del polvo doméstico por Davies (1960), Bronswijk \& Sinha (1973), AbdelHafez et al. (1986) y Dronda (1981). Permanente en nuestros muestreos y sin estacionalidad acusada.

Aspergillus versicolor (Vuill.) Tiraboschi, in Thom \& Church, The Aspergilli: 142-144, 1926.

Citada en polvo doméstico en Canadá, Inglaterra, Francia, Alemania, Paises Bajos, Japón (Rijckaert et al., 1981), Egipto (Abdel-Hafez et al., 1986) y España (Dronda, 1981). Permanente en nuestros muestreos, con los máximos en la primavera.

Aureobasidium pullulans (De Bary) Arnaud, Ann.

Ec. Agric. Montpellier, N.S. 16: 39, 1918.

Causante de esporosis (Hyde, 1972). Aislada de polvo doméstico por Davies (1960), Hirsch \& Sosman (1976) y Dronda (1981). Ocasional en nuestros muestreos.

Botrytis cinerea Pers. ex Nocca \& Balbis, Syst. Mycol. 1: XLV, 1821.

Citada en el polvo doméstico de Londres por Davies (1960), en Holanda por Bronswijk \& Sinha (1973) y en España por Dronda (1981). Sólo hemos aislado una colonia.

Chaetomium Kunce ex Fr., Syst. Mycol. 3: 226, 1829.

Aislado de muestras de polvo doméstico por Davies (1960), Bronswijk \& Sinha (1973), AbdelHafez et al. (1986) y Dronda (1981). Ocasional en los muestreos realizados, sólo hemos podido aislarlo a nivel de género.

Cladosporium cladosporioides (Fr.) de Vries, Contribution to the knowledge of the genus
Cladosporium Link ex Fr.,: 57, 1952.

Citada en el polvo doméstico por Bronswijk \& Sinha (1973), Gravesen (1978), Abdel-Hafez et al. (1986) y Dronda (1981). Frecuente en nuestros muestreos, con máximos durante los meses de octubre a enero.

- Cladosporium macrocarpum Preuss, Sturm's Deut. Fl., 3: 27-28, 1848.

Citada en el polvo doméstico por Gravesen (1978) y Dronda (1981). Sólo la hemos detectado en dos ocasiones.

Cladosporium sphaerospermum Penz, Michelia 2: 473, 1882.

Citada en muestras de polvo doméstico (Abdel-Hafez et al., 1986). Ocasional en nuestros muestreos.

Cunninghamella echinulata (Thaxter) Thaxter, Rhodora 5: 98, 1903.

Ocasional en nuestros muestreos.

\section{Curvularia brachyspora Boedijn.}

No tenemos datos de que esta especie haya sido aislada con anterioridad en nuestro país. Sólo hemos detectado una colonia.

Curvularia lunata (Wakker) Boedijn, Bull. Jard. Bot. Buitenz.13(1): 120-134, 1933.

Aislada de muestras de polvo doméstico en Londres (Davies, 1960). Frecuente en nuestros muestreos y sin estacionalidad.

Curvularia oryzae Bugnicourt.

Creemos que no ha sido aislada con anterioridad en nuestro país. Sólo la hemos encontrado en una ocasión.

Drechslera australiensis (Bugnicourt) Subram \& Jain, Curr. Sci., 35: 354, 1966.

Permanente en nuestros muestreos, con máximos durante los meses de noviembre a febrero.

Drechslera biseptata (Sacc. \& Roum.) Richardson \& Fraser, Trans. Br. Mycol. Soc.51: 148, 1968.

Ocasional en los muestreos realizados, detectándose mayor número de esporas durante el mes de enero. 
Drechslera ravenelii (Curt.) Subram \& Jain, Curr. Sci. 35: 354, 1966.

Sólo la hemos aislado en dos ocasiones.

Emericella nidulans (Eidam) Vuillemin, Compt. rend., 184: 137, 1927.

Estado ascospórico de Aspergillus nidulans (Eidam) Wint., in Rab. Krypt. Fl. 1(2): 62, 1884. Se ha obtenido a partir de muestras de polvo doméstico (Abdel-Hafez et al., 1986). Ocasional en nuestros muestreos y fundamentalmente durante el segundo año.

Eurotium chevalieri Mangin, Ann. Sci. Nat. Bot., Ser. 9, 10: 361-362, 1909.

Estado ascospórico de Aspergillus chevalieri (Mangin) Thom \& Church, The Aspergilli: 111112, 1926.

Citada en muestras de polvo en Holanda (Bronswijk, 1973) y en España (Dronda, 1981). Frecuente en nuestros muestreos, destacando el mes de abril del primer año.

Fusarium Link ex Fr., Syst. Mycol.1: 41, 1821.

Aislado de polvo doméstico por Davies (1960), Bronswijk \& Sinha (1973), Abdel-Hafez et al. (1986) y Dronda (1981). Permanente en nuestros muestreos, con máximos en invierno y principios de la primavera, aunque no presenta clara estacionalidad. Este taxon sólo lo hemos podido determinar a nivel de género.

Geotrichum candidum Link ex Pers., Mycol. Europ., 1: 26, 1822.

Potencialmente patogénico (Alexopoulos \& Mims, 1985). Citada en el polvo doméstico por Horak (1987). Sólo hemos aislado una colonia.

Gliocladium Corda, Icones Fung., 4:30, 1840.

Citado en muestras de polvo doméstico por: Davies (1960), Bronskwijk \& Sinha (1973) y Hirsch \& Sosman (1976). Aislado en una ocasión, sólo lo pudimos identificar a nivel de género.

Monilia sitophila (Montagne) Saccardo.

Citada como alergogénica por Gravesen (1972). Hallada en el polvo doméstico por Bronswijk \& Sinha (1973) y Hirsch \& Sosman (1976). Frecuente en nuestros muestreos, con máximos a principios de invierno.
Mucor circinelloides V. Tieghem, Ann. Sci. Nat. 1: $94,1875$.

Frecuente en nuestros muestreos, con mayor número de esporas en el otoño.

Mucor hiemalis Wehmer, Ann. Mycol. 1: 37, 1903.

Permanente en las muestras obtenidas, con máximos durante los meses de septiembre-octubre.

Mucor plumbeus Bon., Abh. Naturforsch. Ges. Malle 8: 109, 1864.

M. spinosus van Tieghem.

Citada en el polvo doméstico (Gravesen, 1978, 1979). Ocasional en nuestros muestreos.

Mucor racemosus Fres., Beiträge zur Mykologie 1: $12,1850$.

Aislada de polvo doméstico (Davies, 1960; Gravesen, 1978; Abdel-Hafez et al., 1986). Ocasional en los muestreos realizados.

Mycotypha africana Novak \& Backus, Mycologia, 55: 793, 1963.

No tenemos referencias de su aislamiento anterior. Sólo la hemos aislado en una ocasión.

Mycotypha microspora Fenner, Mycologia 24: 196, 1932.

Ocasional en nuestros muestreos.

Myrothecium roridum Tode ex Fr., Syst. Mycol. 3: $217,1829$.

Ocasional en nuestros muestreos.

Myrothecium striatisporum Preston, Trans. Br. Mycol. Soc. 31: 275-276, 1948.

Ocasional en nuestros muestreos.

Myrothecium verrucaria (Alb. \& Schw.) Ditm. ex Fr., Syst. Mycol., 3: 217, 1829.

Citada en el polvo doméstico en Egipto (Abdel-Hafez et al., 1986). Sólo hemos aislado dos colonias.

Nigrospora sphaerica (Sacc.) Mason, Trans. Br. Mycol. Soc. 12: 158, 1927.

Encontrada en polvo doméstico por Bronswijk \& Sinha (1973). Sólo hemos aislado una colonia. 
Paecilomyces carneus (Duché \& Heim). Brown \& Smith, Trans. Br. Mycol. Soc. 40: 70, 1957. Sólo hemos detectado una colonia.

Paecilomyces farinosus (Holm ex. S.F. Gray) Brown \& Smith, Trans Br. Mycol. Soc. 40: 50, 1957.

Ocasional en nuestros muestreos y hallado fundamentalmente durante el primer año.

Paecilomyces variotii Bain., Bull. Soc. Mycol. Fr. 23: 26, 1907.

Esta especie puede causar la denominada paecilomicosis. Aislada de polvo doméstico por Abdel-Hafez et al. (1986). Permanente en nuestros muestreos, presentando varios máximos a lo largo del año.

Penicillium Link ex Gray, Nat. Arr. Br. Pl., 1: 554, 1821.

Citado como un género habitual en el polvo doméstico por Davies (1960), Bronswijk \& Sinha (1973), Hirsch \& Sosman (1976), Gravesen (1978), Abdel-Hafez et al. (1986) y Horak (1987). Aislado en el polvo de colegios por Gravesen et al. (1983, 1986). En España se ha citado su presencia en el polvo doméstico por Ales et al. (1957), JiménezDíaz et al. (1960) y Dronda (1981). En nuestro estudio ocupa el primer lugar por el número de esporas aisladas, pero debido a la complejidad del género sólo hemos podido determinarlo hasta serie (Pitt, 1979), identificándose 14 de ellas. Algunas series presentan una acusada estacionalidad, con máximos generalmente a finales de invierno y en primavera.

Serie Arenicola Pitt, The Genus Penicillium: 394 , 1979. (Ocasional).

Serie Camembertii Raper \& Thom ex Pitt, The Genus Penicillium: 358, 1979. (Ocasional).

Serie Citreonigra Pitt, The Genus Penicillium: 218,1979 . (Ocasional).

Serie Citrina Raper \& Thom ex Pitt, The Genus Penicillium: 290, 1979. (Permanente).

Serie Expansa Raper \& Thom ex Fassatiovà, Acta Univ. Carol., Biol., 12: 324, 1977. (Frecuente).

Serie Fellutana Pitt, The Genus Penicillium: 263, 1979. (Ocasional).

Serie Glabra Pitt, The Genus Penicillium: 169,
1979. (Permanente).

Serie Implicata Raper \& Thom ex Pitt, The Genus Penicillium: 191, 1979. (Ocasional).

Serie Islandica Pitt, The Genus Penicillium: 445, 1979. (Permanente).

Serie Miniolutea Pitt, The Genus Penicillium: 419, 1979. (Ocasional).

Serie Olsonii Pitt, The Genus Penicillium: 392, 1979. (Ocasional).

Serie Oxalica Raper \& Thom ex Pitt, The Genus Penicillium: 273, 1979. (Ocasional).

Serie Restricta Raper \& Thom ex Pitt, The Genus Penicillium: 205, 1979. (Frecuente).

Serie Urticicola Fassatiovà, Acta Univ. Carol., Biol., 12: 324, 1977. (Permanente).

Phoma Sacc., Michelia 2: 4, 1880.

Aislado del polvo doméstico por Davies (1960). Permanente en los muestreos realizados y con un aumento acusado en los meses de primavera. Sólo hemos podido identificarlo a nivel de género.

Rhizopus nigricans Ehrenb. ex Corda, Icon Fung. 2: $20,1838$.

Esta especie ha sido citada en muestras de polvo doméstico por Bronswijk \& Sinha (1973), Gravesen (1978), Abdel-Hafez et al. (1986) y Horak (1987). Permanente en nuestros muestreos y con máximos durante el otoño.

Sartorya fumigata Vuillemin, Fide Benjamin, in Mycologia 47(5): 678, 1955.

Estado ascospórico de Aspergillus ficheri Wehmer, in Centr. Bakteriol. Parasitenk, Abt. II, 18: 390-392. 1907.

Sólo hemos hallado una colonia.

Syncephalastrum racemosun Cohn ex Schroeter, Krypt. Fl. Schles. 3(2): 217, 1886.

Esta especie ha sido aislada de polvo doméstico por Davies (1960) y Bronswijk \& Sinha (1973). Sólo hemos hallado una colonia.

Torula Pers. ex Fr., Syst. Mycol. 3: 499, 1932.

Ocasional en nuestros muestreos. Sólo ha podido ser identificada a nivel de género.

Trichoderma harzianum Rifai, Mycol. Pap. 116: 38, 1969. 
Frecuente en nuestros muestreos, no tenemos referencias de citas anteriores por otros autores en el polvo.

Trichoderma koningii Oud, apud Oud \& Koning, Archs. Néerl. Sci., II, 7: 291, 1902.

Frecuente en nuestros muestreos y con máximos en el mes de febrero.

Trichoderma viride Pers. ex. S.F. Gray aggr., Mycol. Pap. 116: 47, 1969.

Produce micotoxinas que causan severas irritaciones locales, inflamaciones y necrosis de piel, así como problemas en la vista (Abdel-Hafez \& Shoreit, 1985). Citada en el polvo doméstico por Davies (1960), Bronswijk \& Sinha (1973), AbdelHafez et al. (1986), Horak (1987) y Dronda (1981). Sólo hemos hallado una colonia.

Ulocladium alternariae (Cooke) Simmons, Mycologia 59: 82-83, 1967.

Stemphylium alternariae (Cooke) Sacc.

Sólo hemos aislado una colonia.

\section{CONCLUSIONES}

Se han aislado 91 táxones, de los que el $13 \%$ pertenecen a Ascomicetes, el $19 \%$ a Zigomicetes y el $68 \%$ a Deuteromicetes; los más abundantes han sido: Penicillium, Aspergillus, Levaduras, Alternaria, Phoma y Fusarium. Habiéndose aislado un mayor número de colonias de: Aspergillus niger, Aspergillus fumigatus, Alternaria tenuis y Aspergillus terreus. De los táxones aislados el $38 \%$ son considerados potencialmente patogénicos en mayor o menor medida, porcentaje similar al señalado por otros autores para interiores.

Existen diferencias entre los colegios muestreados, respecto al número de colonias dèsarrolladas en ellos, destacando por los aislamientos aquellos colegios que se sitúan próximos al río Guadalquivir o que son edificios antiguos. Se presenta una distribución estacional en la concentración de esporas fúngicas, mostrando un máximo durante los meses de mayo a septiembre. Géneros como Cladosporium, muy frecuente en la micoflora del aire, presentan en el polvo una menor incidencia. De acuerdo con otros autores probablemente debido a pérdida de viabilidad en este hábitat.

AGRADECIMIENTOS. Los autores agradecen el apoyo financiero de la CICyT para la realización de este trabajo como parte del proyecto SM 890010 .

\section{BIBLIOGRAFÍA}

ABDEL-HAFEZ, S.I.I. \& A.A.M. SHOREIT 1985- Mycotoxins producing fungi and mycoflora of air-dust from Taif, Saudi Arabia. Mycopathol., 92: 65- 71.

ABDEL-HAFEZ, S.I.I., A.A.M. SHOREIT, A.I.I. ABDEL-HAFEZ \& O.M.O.E. MAGHARABY -1986- Mycoflora and mycotoxin-producing fungi of air-dust particles from Egypt. Mycopathol., 93: 25-32.

ALES, J.M., G. CANTÓ, L.M. GARCÍA, C. JIMÉNEZ-DIAZ, F. LAHOZ, F. ORTIZ y A. SASTRE -1957- Papel etiológico de los hongos del aire en el asma bronquial. Rev. Clin. Esp., 64(3): 143-152.

ALEXOPOULUS, C.J. \& C.W. MIMS -1985- Introducción a la Micología. Omega. Barcelona. $638 \mathrm{pp}$.

BERNTON, P. -1930- Asthma due to mold Aspergillus fumigatus. J. Amer. Med. Ass., 95: 189-195.

BONILLA-SOTO, D., N.R. ROSE \& C.E. ARBESMAN -1961- Allergenic molds: antigenic and allergenic properties of Alternaria tenuis. J. Allerg., 32: 246-270.

BRONSWIJK, J.E.M.H. -1972- House-dust Ecosystem and House-dust Allergen(s). Act. Allergol., 27: 219-228.

BRONSWIJK, J.E.M.H. -1973- Dermatophagoides pteronyssinus (Trouessart 1897) in mattress and floor dust in a temperate climate (Acari: Pyroglyphidae). J. Med. Entomol., 10: 63-70.

BRONSWIJK, J.E.M.H. \& R.N. SINHA -1973Role of fungi in the survival of Dermatophagoides (Acarina: Pyroglyphidae) 
in house-dust enviroment. Environ. Entomol., 2: $142-145$.

CALVO, M.A., M.A. DRONDA \& R. CASTELLO -1982-Fungal spores in house dust. Ann. Allerg., 49: 213-219.

CALVO, M.A., J. GUARRO y E. VICENTE 1978-Presencia de Aspergillus fumigatus en la atmósfera urbana. Anal. Med. Cir., 251: 69-73.

CIEGLER, A. -1979- Fungi that produce mycotoxins: conditions and occurrence. Mycopathol., 65: 5-11.

CHAKRAVERTY, R. \& S. SINHA -1985- The incidence of Aspergillus parasiticus in the indoor and outdoor environments of Calcuta, India. Grana, 24: 133-135.

D'AMATO, G., G. COCCO, G. RUGGIERO \& L. SALES -1975- Importanza dei miceti nella patologia respiratoria su base allergica. I. Incidenza di positivita ai test cutanei. Arch. Monaldi Tisiol. Malat. Apparat. Respir., 30: 18.

DAVIES, R.R. -1958- Moulds in dust and air. Thesis. Univ. London. 213 pp.

DAVIES, R.R. -1960- Viable moulds in house dust. Trans. Brit. Mycol. Soc., 43(4): 617-630.

DRONDA, M.A. -1981- Estudio de la micoflora presente en el polvo intradomiciliario. Tesina Fac. Farm. Univ. Barcelona.

DUCE, F., S. BELLO, A. VILA, A. REZUETA y C.M. RUBIO -1986- Estudio de los hongos intra y extradomiciliarios en sujetos con hipersensibilidad inmediata en Zaragoza (España). Allergol. Immunopathol., 14: 101-106.

GRAVESEN, S. -1972- Identification and quantitation of indoor airborne micro-fungi during 12 months from 44 Danish homes. Act. Allergol., 27: 337-354.

GRAVESEN, S. -1978- Identification and prevalence of culturable mesophilic microfungi in House-dust from 100 Danish Homes. Allerg., 33: $268-272$.

GRAVESEN, S. -1979- Fungi as a Cause of allergic disease. Allerg., 34: 135-154.

GRAVESEN, S. -1981-On the connection between the occurrence of airborne microfungi and allergy symptoms. Grana, 20: 225-227.

GRAVESEN, S. -1987- Microbial and dust-pollutin in non industrial work places. Adv. Aerobiol. EXS., 51: 279-282.

GRAVÉSEN, S., L. LARSEN, F. GYNTELBERG
\& P. SKOV -1986- Demonstration of microorganisms and Dust in Schools and Offices. Allerg., 41: 520- 525.

GRAVESEN, S., L. LARSEN \& P. SKOV -1983Aerobiology of Schools and public institutions -part of a study. Ecol. Dis., 2(4): 411-413.

GREGORY, P.H. -1973- The Microbiology of the atmosphere. Leonard Hill. $2^{\circ}$ ed. Aylesbury. $337 \mathrm{pp}$.

HIRSCH, S.R. \& J.A. SOSMAN -1976- A one year survey of mold growth inside twelve homes. Ann. Allerg., 36: 30-38.

HORAK, B. -1987- Preliminary study on the concentration and species composition of bacteria, fungi and mites in samples of house dust from Silesia (Poland).Allergol. Immunopathol., 15(3): 161-166.

HYDE, H.A. -1972- Atmospheric pollen and spores in relation to allergy. Clin. Allerg., 2: 153-179.

INFANTE, F. y E. DOMÍNGUEZ - 1987- Incidencia de esporas de Cladosporium Link ex Fr. en los hábitats domésticos de la ciudad de Córdoba. Act. VI Simp. Nac. Bot. Cript., 281-290.

INFANTE, F. \&E. DOMINGUEZ -1988- Annual variation of Cladosporium spores in homes habitats in Córdoba, Spain. Ann. Allerg., 60(3): 256- 261.

INFANTE, F., E. DOMÍNGUEZ, E. RUIZ DE CLAVIJO \& C. GALÁN -1987a- Incidence of Alternaria Nees ex Fr. in dwelling of Córdoba city (Spain). Allergol. Inmunopathol., 15(4): 221-224.

INFANTE, F., E. RUIZ DE CLAVIJO, C. GALÁN \& G. GALLEGO -1987b- Ocurrence of Alternaria Nees ex Fr. in indoor and outdoor habitats in Córdoba (Spain). Adv. Aerobiol. EXS., 51: 157-163.

INFANTE, F., E. RUIZ DE CLAVIJO, C. GALÁN y E. DOMÍNGUEZ - 1987c- Estudio comparativo de Alternaria Nees ex Fr. en el aire de exterior e interior en la ciudad de Córdoba. An. Asoc. Palinol. Leng. Esp., 3: 5-11.

JIMÉNEZ-DÍAZ, C., J.M. ALES, F. ORTIZ, F. LAHOZ, L.M. GARCÍA-PUENTE \& G. CANTÓ -1960- The aetiologic role of molds in bronchial asthma. Act. Allergol., suppl. VII: 139-149.

JOLY, P. -1964- Le genre Alternaria. Paul Lechevalier. Encycl. Mycol., 33. 250 pp.

MERCANTINI, R., R. MARSELLA, L. 
LAMBIASE \& M. BELARDI - 1986- Isolation of Keratinophilic fungi from floors in Roman kindergarten and secondary schools. Mycopathol., 94: 109-115.

MERCANTINI, R., R. MARSELLA, L. LAMBIASE \& F. FULVI -1983- Isolation of keratinophilic fungi from floors in Roman primary schools. Mycopathol., 82: 115-120.

MULLINS, J., R. HARVEY \& A. SEATON -1976Sources and incidence of airborne Aspergillus fumigatus (Fres.). Clin. Allerg. 6: 209-217.

MUÑOZ, F. y M.A. MARTÍN -1983-Alergia a hongos. Publ. Sandoz. Barcelona. 66 pp.

NOVEY, H.S. \& I.D. WELLS -1978- Allergic bronchopulmonary aspergillosis caused by Aspergillus ochraceus. A.J. Case Reports, 70(5): 840-843.

PITT, J.I. -1979- The genus Penicillium and its teleomorphic states Eupenicillium and Talaromyces. Academic Press. London. 635 pp.

PORTUS, M., J. GALLEGO, C. BLASCO, y F. MUÑOZ-LÓPEZ -1977- Factores que influyen en la composición de la fauna de ácaros del polvo doméstico. II Lugar de recogida de la muestra. Allergol. Immunol., 5(2):101-110.

RIJCKAERT, G., J.E.M. BRONSWIJK \& H.F. LINSKENS -1981- House-dust Community (Fungi, mites) in different climatic regions. Oecologia (Berl.), 48: 183-185.

SANDHU, R.S., S.K. MEHTA, Z.U. KHAN \& M.M. SINGH -1978- Role of Aspergillus and Candida species in allergic bronchopulmonary mycoses a comparative study. Mycopathol., 63: 21-28.

SAMSON, R.A. -1985- Occurrence of moulds in modern living and working environments. Eur. J. Epidemiol., 1(1): 54-61.

SEDERBERG-OLSEN, J. \& F. HARDT -1984The indoor climate in schools. Ugeskr Laeger, 146: 1453-1455.

SOLOMON, W.R. -1975- Assessing fungus prevalence in domestic interiors. J. Allerg. Clin. Immunol., 56(3): 235-242.

TORRES J.M., J. MARTÍNEZ, M. COROMINAS y J. ACEBILLO -1982- Identificación y distribución de hongos atmosféricos intra y extradomiciliarios en Barcelona por medio del cultivo en placa. Act. XIII Cong. Nac. SEA. Sevilla.
TUITE, J. -1969- Plant pathologicalmethods fung $i$ and bacteria. Burgess publishing company, USA, $239 \mathrm{pp}$.

WERFF, P.J. -1958- Mould Fungi and Bronchial Asthma. Thomas, Springfield. Illinois. $174 \mathrm{pp.}$

WHO -WORLD HEALTH ORGANIZATION- 1983-Indoor air pollutants: Exposure and health effects. WHO-Meeting in Nördlingen 1982. Eur. Report $N^{\circ} 78$.

Aceptado para su publicación en Febrero de 1993

Dirección de los autores. Departamento de Biología Vegetal y Ecología. Facultad de Ciencias. Avda. San Alberto Magno, s/n. 14004-Córdoba. 